\title{
Modularized Cutting Tool Selection Expert System
}

\author{
Chen Wang ${ }^{1}$, Wu Zhao, ${ }^{* 1}$, Ling Chen ${ }^{2}$, Kai Zhang ${ }^{1}$ and Xin Guo ${ }^{1}$ \\ ${ }^{I}$ School of Manufacturing Science and Engineering, Sichuan University, Cheng Du 610065. China \\ ${ }^{2}$ Division of Production and Materials Engineering, Lund University, Lund, 999027, Sweden
}

\begin{abstract}
This paper is to present a rule-based cutting tool selecting expert system which has knowledge modules and rule bases. Besides, according to different process targets, the selection progress will apply corresponding constraints and rule modules. The logic of tool selection follows a decision-making procedure as an experienced engineers. The strategy of system is to guide the user through several standard steps: information input; feature recognition; selection of machining method; selection of tool material and type; calculation of process parameter and solving cutting problem. This system also has a modularized structure which allows adding new functions and new modules to expand knowledge base and data base. Modules involves in this system are composed of the user interface, knowledge acquisition facility, explanation facility, the knowledge base module, the inference engine and the database module.
\end{abstract}

Keywords: Tool selection, expert system, modularization, process optimization.

\section{INTRODUCTION}

In mechanical manufacture industry, process planning involves scheduling resources, such as machine tools, work piece, cutting tools, operation sequence, processing parameters and the choice of auxiliary functions [1]. During the last 30 years some process planning expert systems have been developed to select tools and cutting parameters for specific operations.

In early stage, process engineers selected adequate cutting parameters with their experience. Obviously, this kind of parameters were selected intuitively considering safety factor. In order to computerize and achieve more accurate cutting process solutions, automation techniques were applied. Then, engineers tried to apply intelligent techniques to optimize of the cutting parameters. Those intelligent techniques includes several optimization methods, such as: genetic algorithms [2] (Cus \& Milfelner 2005), neural networks [3] (Abellan, Romeros, Siller, Estrud, \& Vila, 2008) and bio-inspired techniques [4] (Zain, A. M., Haron, H., \& Sharif, S. 2010). However, these methods provide intrinsic mathematical nonlinear function, hidden in a "black box" giving solutions, on the other hand, it decreases the transparency of process. In the field of manufacturing process planning, expert system involves two steps. First of all, knowledge base system extracts knowledge or experience and then applies fuzzy logic to deduce solutions. As commonly discussed in scientific literature, an online expert system which selecting process information applies fuzzy logic as reasoning mechanism to acquire the knowledge of mechanical engineers [5] (Wong and Hamouda 2003). On the other hand, in order to calculate the cutting parameters and manufacturing route in cutting process, a new computer aided process planning is developed [6] (Vidal, A., Alberti, M., Ciurana, J., \& Casadesus, M. 2005). Moreover, in order to acquire optimal process parameters subject to specific precision and surface quality, tool life expectancy and production time sunder different processing conditions, some theoretical and experimental work has been carried out by organizations in several countries [7-9] (Chien\&Chou, 2001; Vivanco, Luis, Costa, \& Ortiz 2004 and references therein).

These expert systems mainly dealt with the geometrical matters and selection of cutting tools [10]. Most of them concentrated on specific components and realize part function. Some expert systems took the component material and geometric features into consideration, however, ignored the selecting process of the cutting tool type and material. The main aim of this research is to help designers and manufacturing planners to select optimal cutting tools and optimum cutting conditions to reduce cost and time, as well as to solve some technical problems in cutting process with the aid of innovation strategy. The proposed modularized muti-objective cutting process expert system implements the following features, such as component feature recognition; selecting tool material; selecting tool type and optimum cutting conditions and innovative design.

\section{COMPONENTS OF CPES}

Expert system use a large number specific domain knowledge of experts to solve problem [11]. And essentially evolves two kind of terms. One type is constructed with specific kinds of programming languages and tools such as rule-based systems, frame-based systems, and programming languages such as Prolog and LISP [12]. Another type is more appropriate for expert system, because expert system can reasons problem as human expert [13]. This paper tries to propose a classical optimization method integrating innovation strategy, and the design of expert system is based 


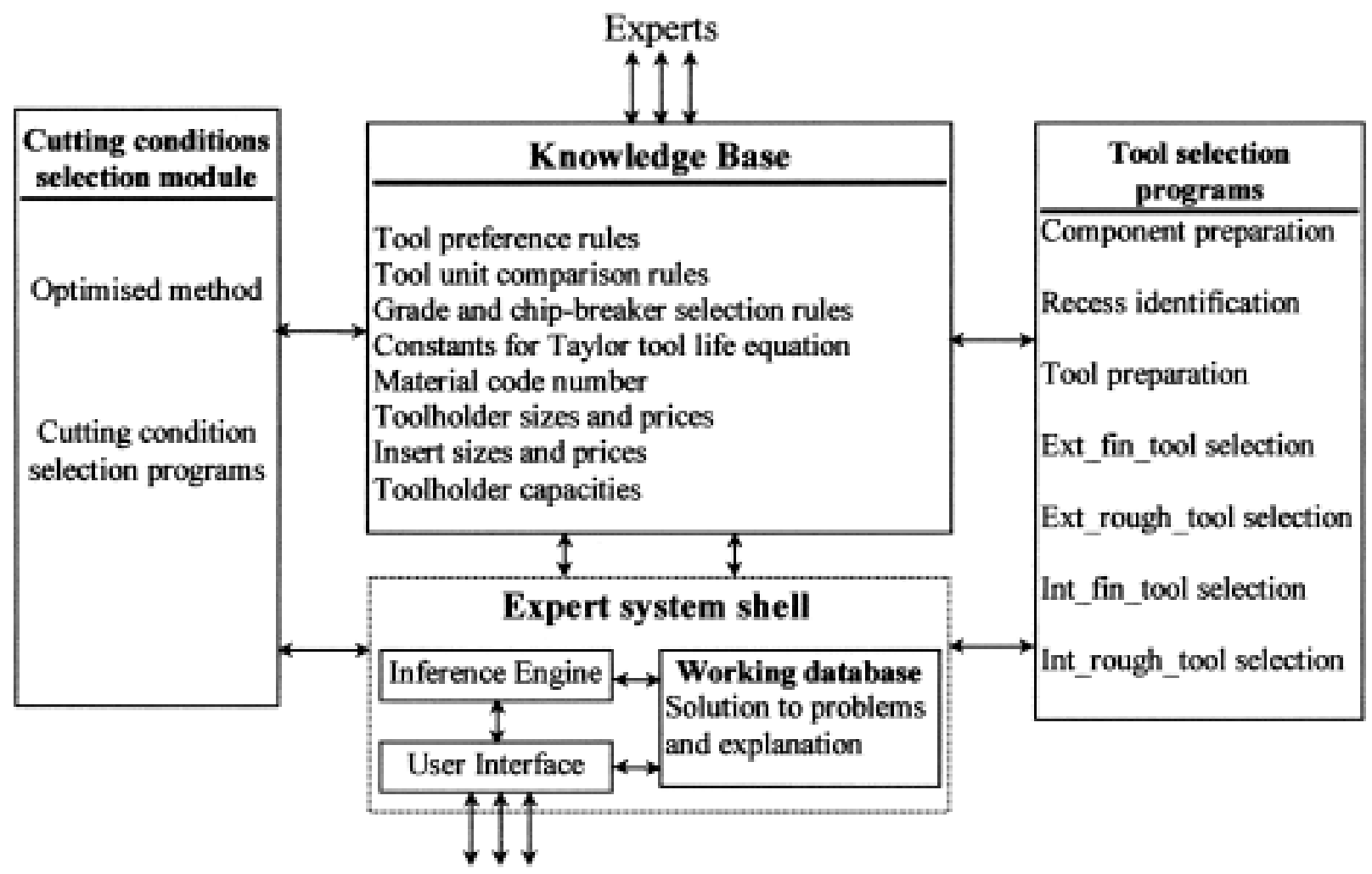

Fig. (1). Structure of the proposed system.

on modularize method. This system also applied theoretical models and semantic method. The proposed expert system involves user interface, inference engine, explanation facility, knowledge acquisition facility, knowledgebase, and working memory and other executable programs. The structure of the proposed expert system is illustrated as Fig. (1).

\subsection{Inference Engine}

The inference engine which manipulates the stored knowledge to reach solutions is one of the basic components of expert system. The inference engine could search knowledge base, and provide answers to user by applying rules to the solution of a particular problem [14]. The scanning process of knowledge will continue until these antecedents match the assertions in database, then user will get the deduced results. Besides, the interpreter provides explanations and can be updated by adding a new separate knowledge base module.

\subsection{Modularized Knowledge Base}

The knowledge of cutting process expert system mainly comes from two sources: experience of domain experts and technical documents [15]. Owning an expandable structure is important for the expansibility of knowledge base, just like domain experts learns new knowledge and applies this knowledge in the future. An independent and modularized knowledge base is proposed, this makes it possible to update the knowledge base by simply input data and editing the knowledge information. The modularized knowledgebase mainly involves factual statements, frames, objects, cases, tables, IF-THEN rules and equations [16]. Knowledge representation technology and rules are the key components of knowledge base. Relevant information about component characteristics and cutting tools in machining process are included in the hierarchy shown as Fig. (2). Elements called 'objects' were presented as frames in hierarchy model. The framework consists of a set of slot which contains a description for the attribute of object. Characteristics of the components, cutting tools and machining process are also expressed as objects which can be classes. The objects relation of the expert system is composed together in a hierarchy building. System identify the characteristics of objects and then apply the appropriate rules to perform the task. Rules in each module will deduce a serious of corresponding conclusions according to correlated conditions. This procedure reflects the logic of knowledge base.

What we want is a knowledge base with fine structure rather than a huge plant knowledge base which includes all the rules. The relevant knowledge in expert system is represented in the terms of the formal logic. The knowledge base contains a sequence of rules in a modular approach. Thus, expert system can be used in modularized way to adapting to different conditions. The proposed knowledge base here includes rules which can be divided into several modules. In each module, rules will be applied according to the proposed approach. These rules can be split into the modules shown as Table $\mathbf{1}$.

\subsection{Database}

Database systems mainly involves two main aspects: data and mathematical model [17]. The cutting tool data about 


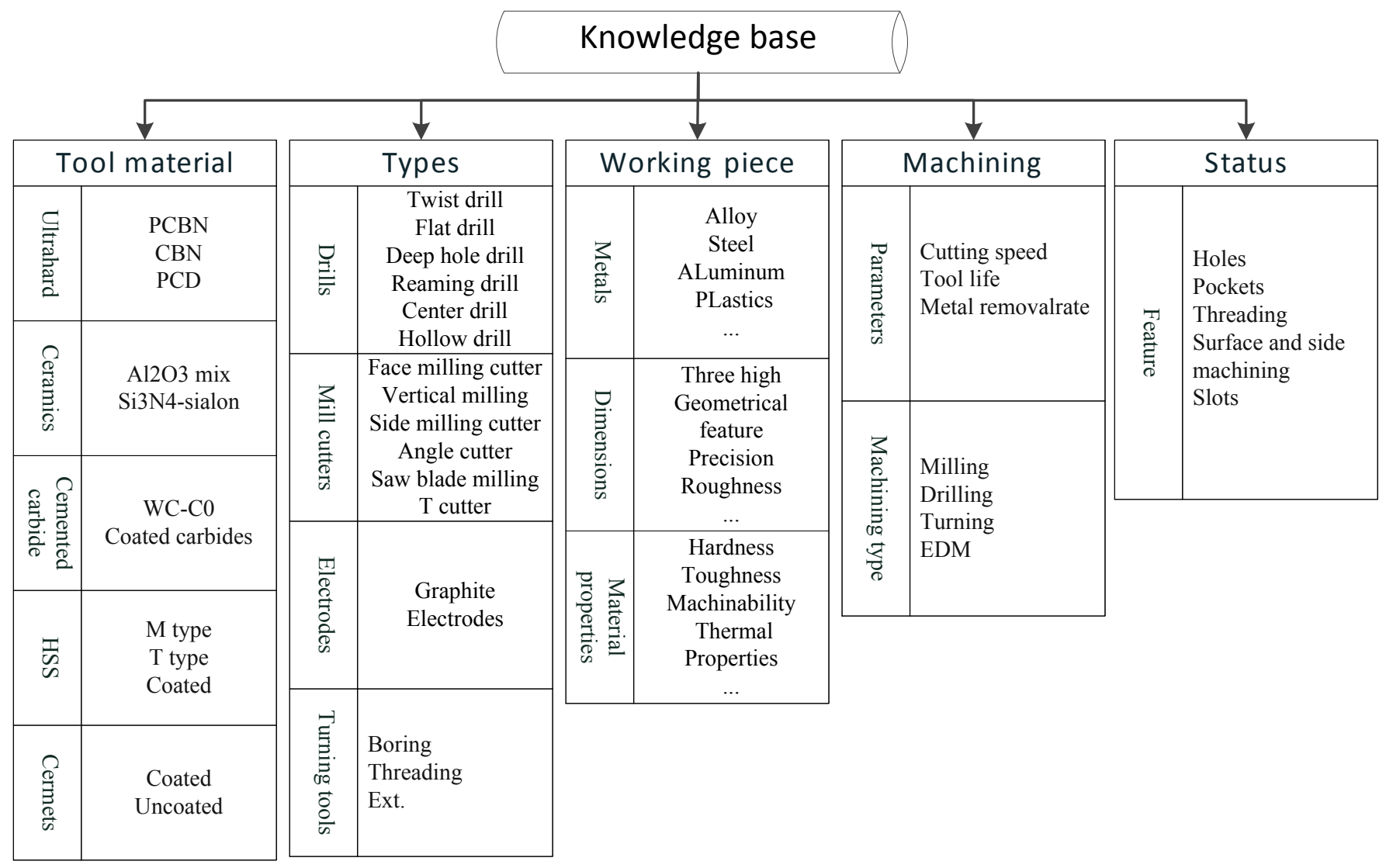

Fig. (2). Hierarchy tree of system.

Table 1. Knowledge modules.

\begin{tabular}{|c|c|c|}
\hline No. & Module & Function \\
\hline \hline I & Stability robustness and allowable & Gives robustness to the system and presents the rough allowable input cutting parameters. \\
\hline II & control of cutting forces & Keeping the forces of the system under prescribed upper limit in spite of variations in system \\
parameters.
\end{tabular}

cutting tool materials, machine tools and work piece materials in catalogues are mainly obtained from the several cutting tool manufactures, large factories and handbooks. The recommended data are put into forms in the database. Mathematical model can be used to calculate the cutting parameters based on the database. The database of system consists of several independent groups of data, shown as Table 2, involving materials, cutting tools, tool materials, cutting parameters, processing method and equations, respectively.

\section{SYSTEM LOGIC}

The cutting process expert system run under an interactive operation environment. Users can describe the characteristic of component with the information integrated into the database. In the process of the operating the software, expert system will guide user through these main steps, including: Inputting information, feature recognition, selecting machining process; selecting tool material; selecting tool type; optimizing cutting conditions and solving problems. The scenario of cutting process expert system is shown as Fig. (3).

\subsection{Process Information Input and Feature Recognition}

The detail information about work piece is the foundation of reasoning process. Under the current technical level, characters identified by engineers have more efficient and 


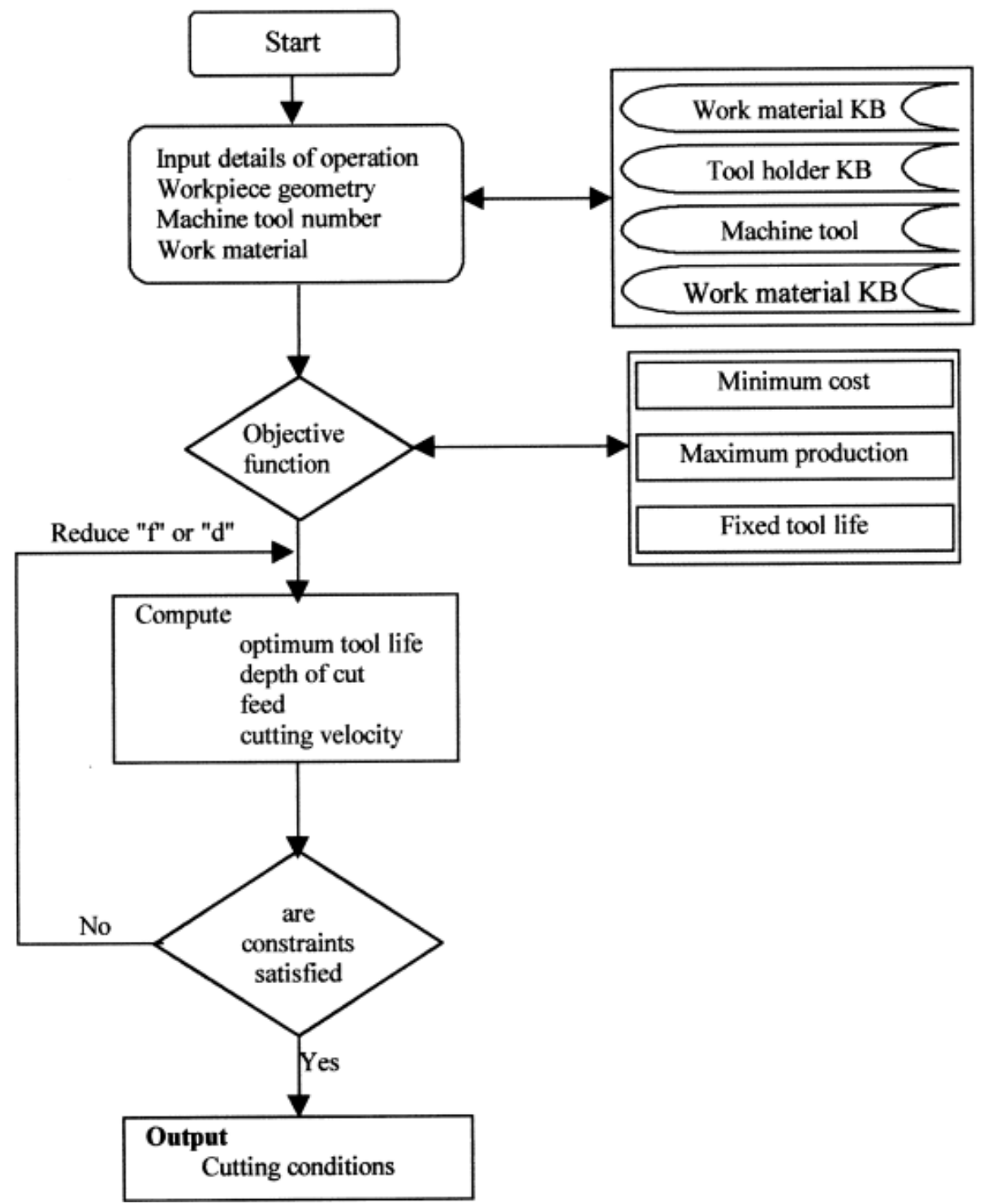

Fig. (3). The scenario of cutting process expert system.

accurate compare to characters identified by computer programs. The identification of piece involves feature specification and dimensions, which need to be entered to system by user. This system provide standardized typical characteristics for user to select. In this stage, system also offer 3D model with typical geometric features for referring. For example, turning surfaces has some of the following characteristics such as face, diameter, arc, recess, grooving and taper, etc. And these characteristics are also be divided as two groups according to the importance, shown as Fig. (4).

\subsection{Selecting Machining Process}

The above information about work piece enables expert system to select the appropriate cutting process and cutting material. The selection machining method need to consider the these factors such as the type, size and precision, surface finishing, dimensional tolerance of the feature. In this stage, system will select the processing methods including

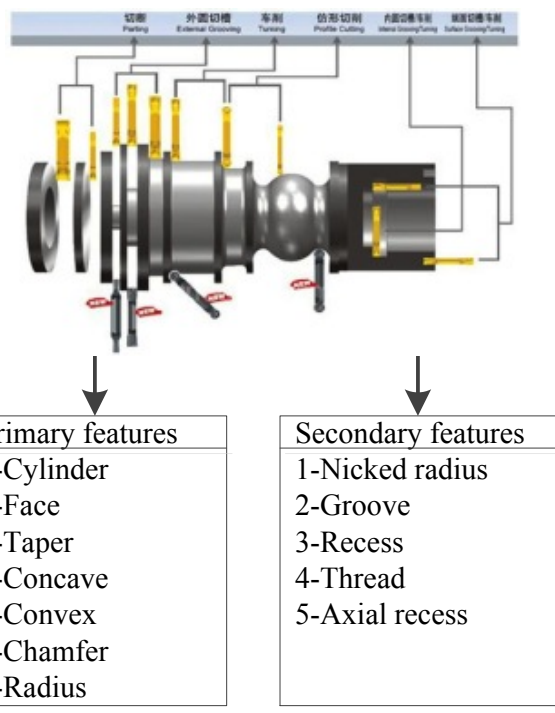

Fig. (4). Feature recognition. 
traditional processing methods such as drilling, turning, milling, etc. and special working technology such as Laser, EDM, etc. The relationship between the aspect ratio and the machining type is shown as Fig. (5). Taking the typical surface finishing process for example, once the feature of curved surface is identified and the parameter is defined, then the system will make judgment.

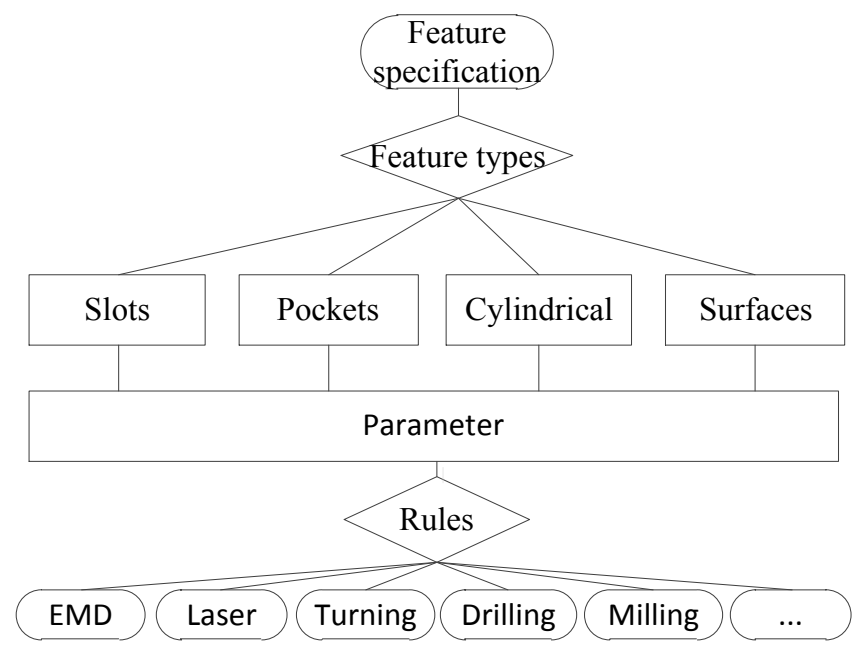

Fig. (5). Corresponding processing method to features.

\subsection{Selecting the Cutting Tool}

The tool selection is the progress of information determination about tool holder, insert, cutting conditions, and coolant. Cutting tools are mainly composed of two parts: tool holder and indexable insert. The selecting sequence is first to select tool holder and then suiting insert. System offer two approaches for selecting cutting tools, manual searching based on the database and automatic selection by system. Manual searching which has more reliability was performed by the user owing abundant of tacit knowledge. The required information for tool selection includes: tool holder (type, clamping system, hand of cut, point angle, size, etc.), insert (size, shape, nose radius, grade, etc.), processing conditions, and coolant. What's more, system will recommend reference case and relevant parameter to user, according to machinability data, feature. The parameters of cutting tools are included as ISO code in table contain the information about the parameters, carbide grades and functions. Selecting tool are carried out through a series of IF-THEN structures to choose the suitable tool holder and insert from the tool library.

The material of cutting tools relies on the material of component to be machined. So it is needed to select the material of component firstly, then CPES will deduce the corresponding tool material. There are three way for use to select component material in this system. One option is that user selects material from the databases stored in expert system. Another way is to browse and select the material from the Cambridge Engineering Selector (CES) linked to expert system. The last option allows users input the material by themselves depending on own experiment and knowledge. Besides, this system provides a serious of rules for selecting suitable tool material corresponding to the selected material of component.

\subsection{Calculating Process Parameters}

The system offers extra function to calculate process parameters such as machining cost and time. The data put in by user can be processed through these equations and rules to calculate parameters. Mathematical stored as rules in knowledge modules are shown in Table 1. Taking the module $\mathrm{V}$ for example, this module is designed to calculate cost and includes serious of equations and rules.

Inference engine can use this cost function to calculate the parameters. For example, the formula (1) can be applied

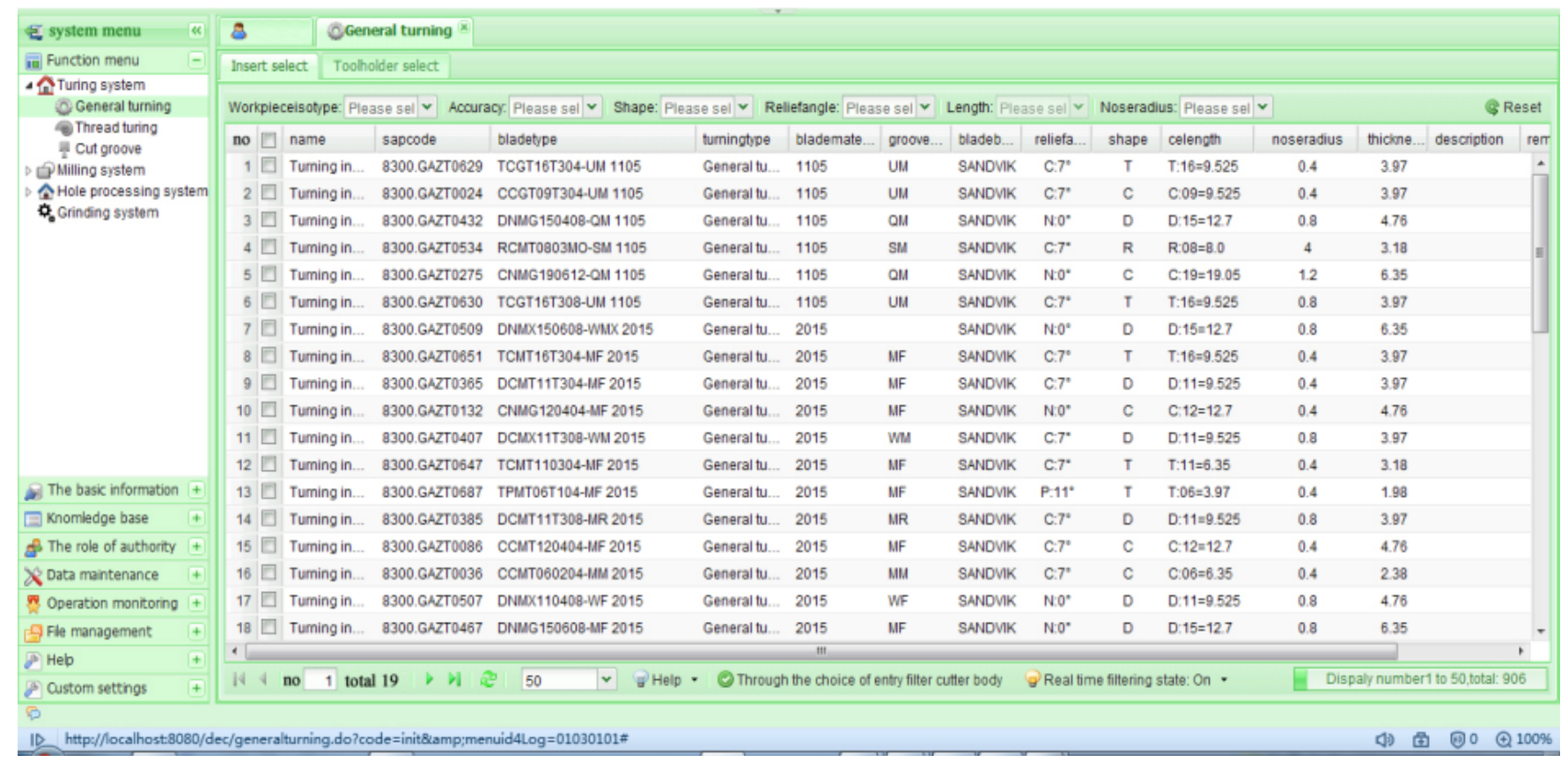

Fig. (6). Selection of tool type. 
to calculate the tool cost of milling, which contains other subfunction, such as TOL: life of the tool; MRR: material remove rate; SURF: surface finish; ROS: robustness of the system. They are defined as following:

$$
\begin{aligned}
& J\left(T O L, M R R, T E S, R, C_{i(i=1, \ldots 4)}\right)= \\
& c_{1} \cdot N F_{1} \cdot T O L+C_{2} \cdot N F_{2} \cdot M R R+C_{3} \cdot \frac{N F_{3}}{S U R F}+C_{4} \cdot N F_{4} \cdot R O S
\end{aligned}
$$

where ci is weighting factor, $N F_{1}$ represents normalisation factor.

$$
T O L=K t o l \cdot V^{-\alpha_{1}} \cdot \alpha_{d c}{ }^{-\alpha_{2}} \cdot f_{t}^{-\alpha_{3}}
$$

where Ktol is model constant, $\mathrm{V}$ is cutting speed ( $\mathrm{m} / \mathrm{min}), \alpha \mathrm{i}$ is model parameter, adc represents axial depth of cut ( $\mathrm{mm}$ )and $\mathrm{ft}$ represents feed per tooth ( $\mathrm{mm} /$ tooth).

$$
\operatorname{MRR}=a_{d c} \cdot r_{d c} \cdot f_{c}
$$

where $r_{d c}$ represents radial depth of cut $(\mathrm{mm})$ and $\mathrm{fc}$ represents feed velocity $(\mathrm{mm} / \mathrm{s})$.

$S U R F=K_{\text {surf }} \cdot V^{\beta_{1}} \cdot f_{c}^{\beta_{2}} \cdot \alpha_{d c}^{\beta_{3}}$

where $K_{\text {surf }}$ is a model constant and, bi represents surface roughness.

$\operatorname{ROS}=\min \left(\operatorname{sqrt}\left\{\left(\alpha_{d c, R O S}\right)^{2}+\left(S_{S, R O S}\right)^{2}\right\}\right)$

where Ss, ROS is the length of the spindle speed.

$$
\begin{aligned}
& N F i=J_{i}-J_{\max } / J_{\text {max }}-J_{\text {miin }} \\
& J=\sum_{j=1}^{4} C_{i} \cdot N F_{i} \cdot J_{i}
\end{aligned}
$$

Besides, there are some more simple rules to estimate process time and cost.

$$
P t=V_{f} / R_{m r}
$$

where $\mathrm{Pt}(\mathrm{min})$ is process time, $\mathrm{Vf}\left(\mathrm{mm}^{3}\right)$ is form feature volume, $\mathrm{Rmr}\left(\mathrm{mm}^{3} / \mathrm{min}\right)$ is material removal rate. The equations and rules of estimated cutting time and feature cost are shown as below:

Set Value ((Processing: RPMd), 256.4* Processing: SPd mmmin ${ }^{-}$ $1 /$ Features: Di mm);

Set Value ((Processing: FRdmmin $\left.{ }^{-1}\right)$;

Processing: frdmmrev ${ }^{-1} *$ Processing: RPMd);

Set Value ((Processing: CTD min., Features: De $\mathrm{mm} /$ Machining: FRd $\left.\mathrm{mmmin}^{-1}\right)$;

Set Value ((Processing: Cost MY), Processing: CTM min *18);

Set Value ((Processing: Cost D $¥)$, Processing: CTD min*18);

Set Value ((Processing: Cost TҰ), Processing: CTT min*18); IF

(Processing cost: drilling $<$ Processing cost: milling)

And (Processing cost: drilling $<$ Processing cost: Turning)

THEN

$$
\text { \{'c:ma. wks", ; }
$$

Read cell $(1,1)$;

Closefile ("c;ma.wks"); \};

Machining Process is drilling.

\subsection{Modify Parameters and Solving Cutting Problems}

In this section, a problem solving module is developed, which can provide two approaches for user to modify the cutting parameter. One way is to offer typical cutting problems for reference, another option is to guide the thinking of user in systematic innovation strategy to generate solutions. The typical problems have been classified into several groups which will be presented to the user according to the design phase. The typical problems mainly come from various tool manufacturers. In this approach, user can view the problem definition option, which displays the problem, possible cause and recommended remedy. Then user can modify the cutting data.

Most cutting problems are caused by unsuitable cutting parameters. So, user needs to check the cutting data firstly with the ideal data calculated by system to find out whether the cutting parameters are correct. If the values of cutting data are match with the ideal data, user should to revise the cutting data. As shown in Fig. (6), the cutting parameter for turning of steel at medium velocity are evaluated, and because the parameter about hardness and tool life is lower than the ideal parameter calculated by system, a warning is given to user Fig. (7).

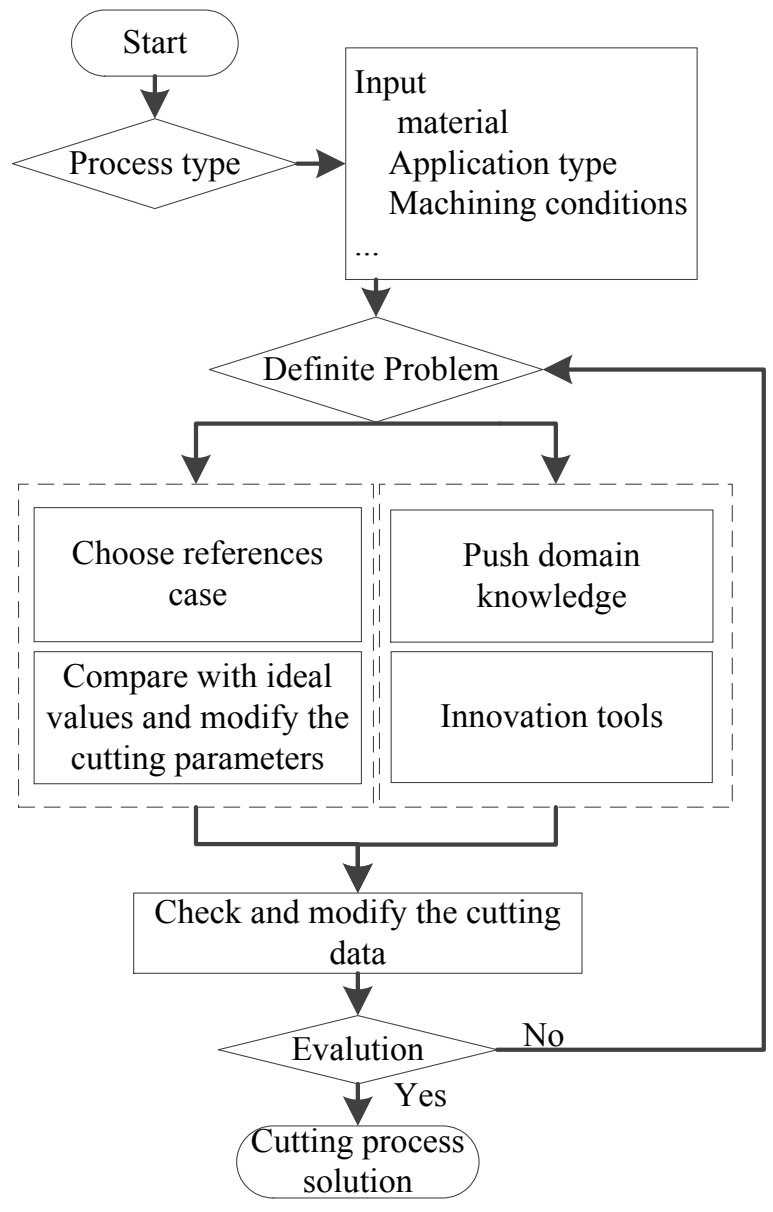

Fig. (7). The logic of modify parameters. 


\section{CONCLUSION}

In this paper, a modularized multi-objective cutting process expert system were developed. This system enables user to select cutting tools and define cutting parameters, with the goal of short time and low cost, etc. It offer a userfriendly interface to users as well as desired results. The modularized multi-objective cutting process expert system composed of several units: the user interface; knowledge acquisition facility; explanation facility; the knowledge base module; the inference engine and the database module. The prototype system was developed based on SQL and Java. System presents the knowledge in a frame format and can complete reasoning based on rules. The strategy of system is guiding user through several steps: information input; feature recognition; selection of machining method; selection of tool material and type; calculation of process parameters and solving cutting problem. Although the prototype was developed, there were insufficient with the ability such as recognition of geometric feature and insufficient of knowledge which need further research.

\section{CONFLICT OF INTEREST}

We confirm that there are no conflicts of interest associated with this publication and all the financial support for this work have been declared in acknowledgement.

\section{ACKNOWLEDGEMENTS}

This work was supported in part by NSFC (National Natural Science Foundation of China) under Grant No.51175357, and Sichuan Province Science and technology support program under Grant No. 2014GZ0114. Gratitude is also extended to the reviewers and the Editor for their valuable comments.

\section{REFERENCES}

[1] A. Oral, and M. C. Cakir, "Automated cutting tool selection and cutting tool sequence optimisation for rotational parts," Robotics and Computer-Integrated Manufacturing, vol. 20, no. 2, pp. 127141, 2004.

[2] F. Cus, and J. Balic, "Optimization of cutting process by GA approach," Robotics and Computer-Integrated Manufacturing, vol. 19, no.1, pp. 113-121, 2003.

[3] J. V. Abellan, F. Romero, H. R. Siller, A. Estruch, and C. Vila, "Adaptive control optimization of cutting parameters for high quality machining operations based on neural networks and search algorithms," Advances in robotics, automation and control. I-Tech Education and Publishing, Austria, pp. 472-491, 2008.

[4] A. M. Zain, H. Haron, and S. Sharif, "Application of GA to optimize cutting conditions for minimizing surface roughness in end milling machining process," Expert Systems with Applications, vol. 37, no.6, pp. 4650-4659, 2010.

[5] S. V. Wong, and A. M. S. Hamouda, "Machinability data representation with artificial neural network," Journal of Materials Processing Technology, vol. 138, no.1, pp.538-544, 2003.

[6] A. Vidal, M. Alberti, J. Ciurana, and M. Casadesus, "A decision support system for optimising the selection of parameters when planning milling operations," International Journal of Machine Tools and Manufacture, vol. 45, no.2, pp. 201-210, 2005.

[7] W. T. Chien, and C. Y. Chou, "The predictive model for machinability of 304 stainless steel," Journal of materials processing technology, vol. 118, no.1, pp.442-447, 2001.

[8] A. Iqbal, N. He, L. Li, and N. U. Dar, "A fuzzy expert system for optimizing parameters and predicting performance measures in hard-milling process," Expert Systems with Applications, vol.32, pp. 1020-1027, 2007.

[9] S. R. Maity, P. Chatterjee, and S. Chakraborty, "Cutting tool material selection using grey complex proportional assessment method," Materials \& Design, vol. 36, pp. 372-378, 2012.

[10] Y. Altintas, "Manufacturing automation: metal cutting mechanics, machine tool vibrations, and CNC design," Cambridge university press, 2012.

[11] N. Mandal, B. Doloi, B. Mondal, and R. Das, "Optimization of flank wear using Zirconia Toughened Alumina (ZTA) cutting tool: Taguchi method and Regression analysis," Measurement, vol. 44, no.10, pp. 2149-2155, 2011.

[12] J. Balic, F. Cus, B. Vaupotic, M. Hijjawi, Z. Bandar, K. Crockett, and D. Mclean, "Intelligent automatic cutting-tool selections for turning operations," In: International Conference on Artificial Intelligence and Machine Learning, Dubai, United Arab Emirates, AIML-11, vol.7, 2011.

[13] A. R. Yildiz, "Cuckoo search algorithm for the selection of optimal machining parameters in milling operations," The International Journal of Advanced Manufacturing Technology, vol. 64, no.1-4, pp.55-61, 2013.

[14] M. Nalbant, H. Gökkaya, and G. Sur, "Application of Taguchi method in the optimization of cutting parameters for surface roughness in turning," Materials \& design, vol. 28, no.4, pp.1379$1385,2007$.

[15] K. V. Wong, and L. O. De, "Applications of nanofluids: current and future," Advances in Mechanical Engineering, 2010.

[16] A. R. Yildiz, "Cuckoo search algorithm for the selection of optimal machining parameters in milling operations," The International Journal of Advanced Manufacturing Technology, vol. 64, no.1-4, pp. 55-61, 2013.

[17] T. Boutros, and M. Liang, "Detection and diagnosis of bearing and cutting tool faults using hidden Markov models," Mechanical Systems and Signal Processing, vol. 25, no. 6, pp. 2102-2124, 2011. 